\title{
Probing photo-carrier collection efficiencies of individual silicon nanowire diodes on a wafer substrate $\uparrow$
}

\author{
S. W. Schmitt, ${ }^{\text {a }}$ G. Brönstrup, ${ }^{\text {ab }}$ G. Shalev, ${ }^{a}$ S. K. Srivastava, ${ }^{\text {ac }}$ M. Y. Bashouti, ${ }^{a}$ \\ G. H. Döhler ${ }^{a}$ and S. H. Christiansen ${ }^{a b}$
}

Vertically aligned silicon nanowire (SiNW) diodes are promising candidates for the integration into various opto-electronic device concepts for e.g. sensing or solar energy conversion. Individual SiNW p-n diodes have intensively been studied, but to date an assessment of their device performance once integrated on a silicon substrate has not been made. We show that using a scanning electron microscope (SEM) equipped with a nano-manipulator and an optical fiber feed-through for tunable (wavelength, power using a tunable laser source) sample illumination, the dark and illuminated current-voltage (I-V) curve of individual SiNW diodes on the substrate wafer can be measured. Surprisingly, the $1-V$-curve of the serially coupled system composed of SiNW/wafers is accurately described by an equivalent circuit model of a single diode and diode parameters like series and shunting resistivity, diode ideality factor and photocurrent can be retrieved from a fit. We show that the photo-carrier collection efficiency (PCE) of the integrated diode illuminated with variable wavelength and intensity light directly gives insight into the quality of the device design at the nanoscale. We find that the PCE decreases for high light intensities and photocurrent densities, due to the fact that considerable amounts of photo-excited carriers generated within the substrate lead to a decrease in shunting resistivity of the SiNW diode and

Received 7th March 2014 Accepted 3rd April 2014

DOI: $10.1039 / c 4 n r 01258 e$

www.rsc.org/nanoscale deteriorate its rectification. The PCE decreases systematically for smaller wavelengths of visible light, showing the possibility of monitoring the effectiveness of the SiNW device surface passivation using the shown measurement technique. The integrated device was pre-characterized using secondary ion mass spectrometry (SIMS), TCAD simulations and electron beam induced current (EBIC) measurements to validate the properties of the characterized material at the single SiNW diode level.

\section{Introduction}

SiNWs are versatile building blocks for highly innovative optoelectronic devices. ${ }^{\mathbf{1 , 2}}$ It has been shown that they can successfully be integrated into devices such as sensors, NW transistors and solar cells of which some are already beyond the prototype stage. ${ }^{3-9}$ Once a silicon $\mathrm{p}-\mathrm{n}$ diode is reduced to a single NW, its opto-electronic characteristics can significantly change due to an enhanced contribution of the electrical surface response, a strong current confinement within the structure and resonant interaction with light. ${ }^{\mathbf{1 0 - 1 3}}$ These phenomena require in-depth understanding in order to successfully replace parts of today's technologies by advantageous NW building blocks. To date,

\footnotetext{
${ }^{a}$ Max Planck Institute for the Science of Light, Günther-Scharowsky-Str. 1, 91058 Erlangen, Germany.E-mail: sebastian.schmitt@mpl.mpg.de

${ }^{b}$ Helmholtz-Zentrum Berlin für Materialien und Energie, Kekuléstrasse 5, 12489 Berlin, Germany

${ }^{c}$ CSIR-National Physical Laboratory, New Delhi-110012, India

$\dagger$ Electronic supplementary information (ESI) available. See DOI: 10.1039/c4nr01258e
}

opto-electronic parameter retrieval of single SiNW diodes is usually performed on individual model structures, where the SiNWs are removed from a substrate (thereby they are taken out of an ensemble of NWs) and electrically contacted using elaborate clean room methods such as e.g. electron beam lithography. ${ }^{\mathbf{1 4 - 1 6}}$

These contacting techniques are time-consuming and of prototyping nature and do most importantly not account for interface resistance and currents flowing between the SiNW and the substrate. However, the cross-talk between the SiNW and its substrate alters the entire opto-electronic picture, so that measured device characteristics of individual SiNW diodes cannot in any way permit the forecast of the behavior of integrated NW devices composed of several to several billions of NWs that act as a device ensemble. To clarify the electrical interaction between substrate and SiNW diode at the level of an individual NW diode with the potential to predict the behavior of the integrated NW based device, vertically aligned SiNW diodes with an axial p-n-junction were fabricated on a Si wafer. Individual SiNW diodes are pre-characterized using secondary ion mass spectrometry (SIMS) to find the location of the 
metallurgical $\mathrm{p}-\mathrm{n}$ junction and TCAD simulations and to simulate its internal electronic structure. Simulations show the location of the depletion region inside the SiNW depending on details of the $\mathrm{p}-\mathrm{n}$-junction doping. Using electron beam induced current (EBIC) we find the origin of carriers in the device under radiative carrier excitation to be in the SiNW in an NW ensemble and in the substrate underneath. ${ }^{17}$

In the study, we show measurements of $I-V$ curves of individual straight, doped axial SiNW diodes with an axial p-njunction in a SiNW diode array residing on a Si substrate wafer. Therefore, we contact individual SiNWs using a tungsten (W) needle mounted to a nano-manipulator inside an SEM, which is equipped with a fiber feed-through for laser illumination with tunable intensity and wavelength. Measurements of the $I-V$ curves with varied illumination show that the serially coupled system SiNW/Si-wafer can be described with the equivalent circuit of an individual diode, so that the diode parameters like shunting resistivity, series resistivity and photocurrents can be derived from a fit. The paper shows that the PCE of the individual diode is strongly dependent on the wavelength and intensity of the illumination, and that these dependences reflect on the performance of the device at the nanoscale. The PCE for shorter wavelengths permits monitoring the effectiveness of the device surface passivation, whereas a reduced PCE for higher illumination intensities points towards a decrease in diode rectification for higher photocurrents that are predominantly contributed by carriers originating from the substrate. In fact, the in-depth assessment of diode parameters shows that high light intensities and photocurrent densities lead to a decrease in the shunting resistivity and a substantial increase of the diode ideality factor of the SiNW diode.

\section{Device fabrication and pre- characterization}

A silicon wafer (float zone, p-type, boron (B) doped, $10^{16} \mathrm{~cm}^{-3}$ ) was spin-coated with a phosphorus (P) spin-on dopant (SOD/ P509, Filmtronics, USA) and annealed at $900{ }^{\circ} \mathrm{C}$ for $30 \mathrm{~min}$. Simultaneously, an ohmic back contact was obtained by the diffusion of a sputter deposited Al layer on the back of the wafer. After removal of SOD and excess Al in a short HF dip (HF 5\%, $30 \mathrm{~s}$ ), the P-diffusion profile in the silicon wafer was recorded using secondary ion mass spectrometry (SIMS). Fig. 1 shows that compensation between $\mathrm{P}$ and $\mathrm{B}$ is reached in a depth of about $700 \pm 40 \mathrm{~nm}$ (metallurgical junction). The wafer with a planar $\mathrm{p}-$ $\mathrm{n}$ junction was subsequently used for the fabrication of SiNWs. To fabricate SiNWs, polystyrene (PS) spheres were used as a hard mask for subsequent patterning using reactive ion etching (RIE) as described in a previous paper in more detail. ${ }^{18}$ The SiNW diameters are strictly determined by the PS-sphere diameters, whereas the SiNW lengths depend on the RIE etching details. With this large area nano-patterning method, an array of $2.3 \pm$ $0.1 \mu \mathrm{m}$ long SiNWs with a diameter of $737 \pm 21 \mathrm{~nm}$ and $1 \mu \mathrm{m}$ pitch was formed. Fig. 1 shows an oblige SEM image of an individual as-etched SiNW for which the red line indicates the position of the metallurgical junction as determined by SIMS

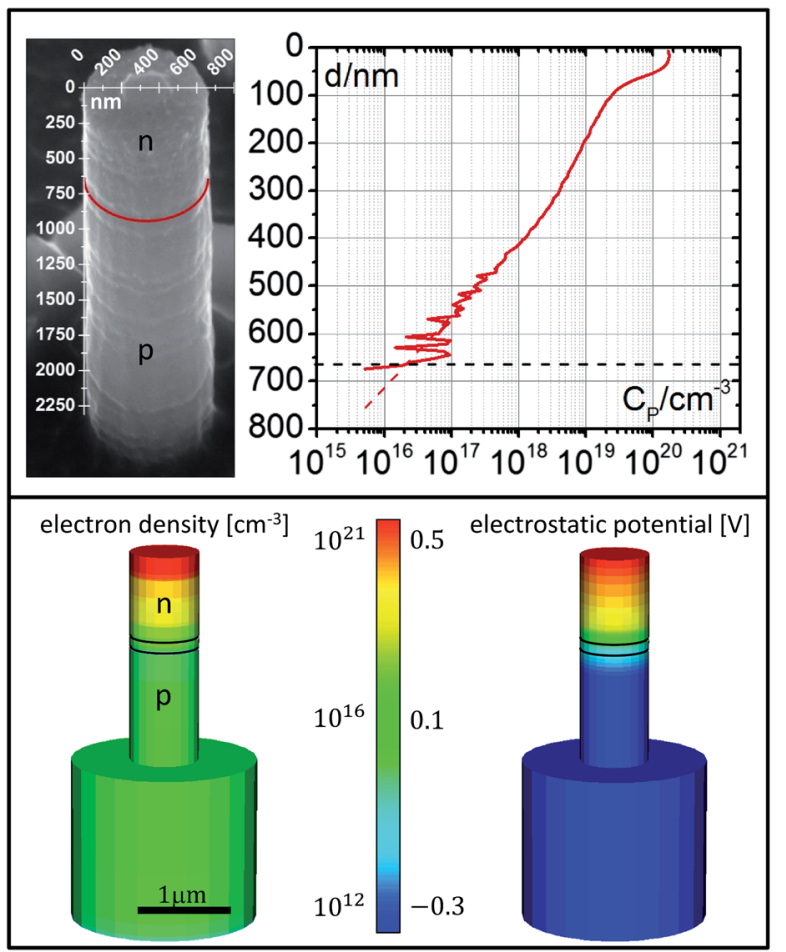

Fig. 1 Top right: SIMS analysis showing the phosphorous diffusion profile in the Si wafer; top left: SEM image of a SiNW in oblique view $\left(45^{\circ}\right.$ tilt). The red line indicates the location of the metallurgical junction; bottom: 3D TCAD simulation showing color coding of the electron density and the electrostatic potential in the SiNW and the underlying Si substrate. The black lines delineate the depletion region determinated by the TCAD simulation.

measurements on the un-patterned P-diffused overall borondoped Si wafer. At the direct contact area between PS spheres and Si wafer, a small circular nap sticks out of the NW surface due to not having been exposed to RIE etching at all as can be seen after removal of the PS spheres in an ultrasonic bath. A device simulator (Synopsys TCAD Sentaurus, Mountain View, CA, USA) was used to determine the electron density and the electrostatic potential in the SiNW diodes (Fig. 1/bottom). Poisson and continuity equations were solved by finite difference time domain (FDTD) calculations in line with the P-diffusion profile measured by SIMS and assuming a configuration in the dark (i.e. zero carrier generation) with no voltage bias between top and bottom contact of the SiNW. The simulations demonstrate that the top part of the SiNW is degenerate (i.e. the minority carrier/electron density is as high as $10^{21} \mathrm{~cm}^{-3}$ ), and that the depletion region (i.e. the electronic $\mathrm{p}-\mathrm{n}$ junction) is clearly located inside the $2.3 \pm 0.1 \mu \mathrm{m}$ long SiNW.

To contact individual SiNWs, a W-needle was mounted onto a nano-manipulator stage inside the SEM (TESCAN Lyra 3). Fig. 2a shows the probing of a free standing SiNW with no neighboring SiNWs due to a local inhomogeneity in the SiNW ensemble. In Fig. $2 \mathrm{~b}$ a SiNW in the NW ensemble was chosen for probing. In both cases the EBIC mappings ( $3 \mathrm{kV}, 100 \mathrm{pA})$ were superimposed (blue) on the SEM images, showing very different EBIC signals from the two essentially identical SiNWs. ${ }^{17}$ The intensity of the EBIC signal depends on the amount of carriers 


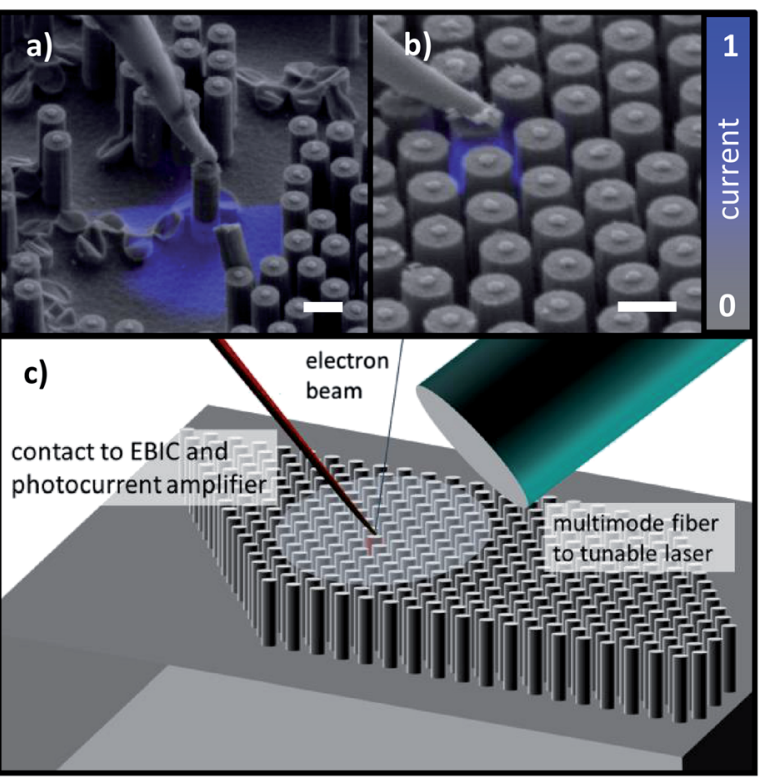

Fig. 2 ( $a$ and b) SEM images of individually contacted SiNWs $\left(45^{\circ}\right.$ tilt angle). The EBIC mappings are superimposed in blue color (scale bars 1 $\mu \mathrm{m})$. (c) Scheme of the experimental setup showing the sample, the Wneedle for contacting and the multimode fiber for tunable laser light illumination.

generated by the incident electron beam and their ability to reach the diffused $\mathrm{p}-\mathrm{n}$ junction located inside the SiNW. Moreover, the EBIC signals are normalized to the maximum intensity in each image, so that the resulting EBIC currents can only serve as a qualitative measure. In Fig. 2 a the EBIC signal at the bottom of the SiNW and in the substrate is the highest and vanishes in the upper part of the SiNW. This can be explained as follows: due to the low beam energy $(3 \mathrm{kV})$ selected for this EBIC measurement, carriers are generated close to the surface $(<150$ $\mathrm{nm}$ depth as obtained from Monte-Carlo-simulations using the SRIM freeware software package, https://www.srim.com) and are therefore strongly affected by recombination of the asetched SiNW surfaces (see an X-ray photoelectron spectroscopy (XPS) scan in the ESI†). This results in an overall lower effective carrier concentration in the SiNWs as compared to the bulk silicon wafer counterpart which has a lower surface to volume ratio. Fig. 2 b shows a SiNW in a densely packed SiNW array, contacted by the W-needle. Due to the low penetration depth of the selected $3 \mathrm{kV}$ electrons these are all absorbed in the SiNWs themselves, i.e. the entire EBIC signal is generated within the contacted NW. The two representative EBIC analyses in Fig. 2a and $\mathrm{b}$ show the origin of the generated current depending on details of the carrier exciting radiation. Depending on the penetration depths of incident electrons (or light, see next section), the overall EBIC current contains contributions from the SiNW alone or the underlying Si substrate in addition.

\section{Measurements and discussion}

To monitor the optoelectronic characteristics of a single axial SiNW diode on a wafer, the device was illuminated with well defined, tunable laser light, incident under an angle of $45^{\circ}$ to the SiNW long axis. $56 \mathrm{I}-\mathrm{V}$ measurements of a single SiNW diode were recorded using illumination with seven different wavelengths $(500,550,600,650,700,750$ and $800 \mathrm{~nm})$ where for each wavelength the light intensity was varied in eight discrete steps $(0,20,50,100,200,500,1000$ and $2000 \mathrm{nW})$. The measurement setup inside the SEM is depicted in Fig. 2c. A multimode glass fiber was transferred to the SEM chamber and was connected to a tunable laser light source outside of the SEM. The light was generated by a white light laser source equipped with a mode-selector box (NKTphotonics). Thereby the sample could be illuminated with (non-polarized) light of controlled wavelengths and intensities. The light intensity at the fiber end was calibrated using a beam splitter outside of the SEM chamber combined with a photodiode. Varying the laser pumping power and the power attenuating filters, the adjustment of distinct intensities at the seven wavelengths of choice was possible. For each intensity-wavelength pair the accurate output intensity at the fiber end was recorded using a calibrated diode. Underneath the fiber, the individual SiNW diodes were contacted as shown in Fig. $2 \mathrm{a}$ and $\mathrm{b}$. To obtain experimental conditions resembling that of an axial $\mathrm{p}-\mathrm{n}$ junction SiNW photovoltaic device under solar illumination, the following measurements were performed using a contact configuration as shown in Fig. $2 b$.

In Fig. 3 the experimentally measured $I-V$ curves for all eight light intensities at a constant wavelength of $700 \mathrm{~nm}$ (example chosen, full dataset see ESI $\dagger$ ) are shown. The superimposed fits were determined by a simulated annealing algorithm. ${ }^{19}$ They are based on a single diode equivalent circuit shown in Fig. 3 (inset) which can analytically be described by the implicit equation

$$
I_{\mathrm{m}}=I_{\mathrm{p}}-I_{\mathrm{D}}-\frac{U+I_{\mathrm{m}} R_{\mathrm{s}}}{R_{\mathrm{p}}}
$$

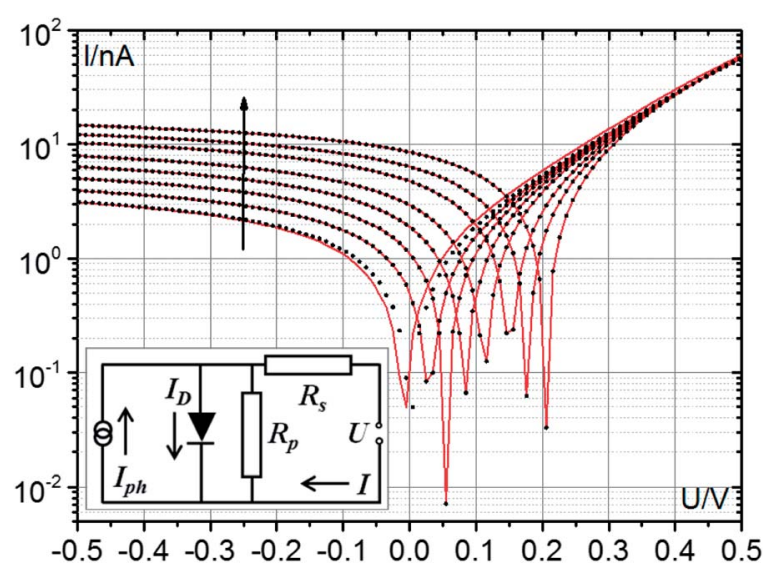

Fig. 3 Black dots show the $I-V$ curves of a single SiNW diode under illumination with $700 \mathrm{~nm}$ (semi-logarithmic scale). The black arrow shows the direction of increasing power in 8 discrete steps at the fiber end inside the SEM. Red lines indicate a fit of the $I-V$ curves with eqn (1). The inset depicts an equivalent circuit of the individually contacted SiNW diode which is analytically described by eqn (1). 
where $I_{\mathrm{m}}$ is the current measured at the load, $U$ is the voltage drop at the load, $I_{\mathrm{p}}$ is the generated photocurrent, $R_{\mathrm{s}}$ and $R_{\mathrm{p}}$ are the series and parallel resistances of the device and $I_{\mathrm{D}}$ is the diode current. $I_{\mathrm{D}}$ is expressed as:

$$
I_{\mathrm{D}}=I_{\mathrm{s}}\left(\mathrm{e}^{\frac{U+I_{\mathrm{m}} R_{\mathrm{s}}}{n k_{\mathrm{B}} T}}-1\right)
$$

where $n$ is the diode quality factor, $I_{\mathrm{S}}$ is the reverse saturation current, $k_{\mathrm{B}}$ is the Boltzmann constant and $T$ is the temperature. For $R_{\mathrm{p}} \rightarrow \infty, R_{\mathrm{S}} \rightarrow 0$, eqn (1) reduces to the diffusion modulated ideal Shockley equation without an implicit dependence on $I_{\mathrm{m}} \cdot{ }^{20}$ Note that the SIMS profile and TCAD simulations (Fig. 1) suggest a degenerated top part of the SiNW and hence an ohmic contact between the $\mathrm{W}$ probe and the SiNW is expected. Therefore, and from the high matching quality of the fits with respect to measurements, it can be concluded that the system SiNW diode/wafer physically resembles a single diode. To permit a judgment of the quality of the fits, all plots are shown in the ESI $\dagger$ both in linear and in semi-logarithmic scale.

The fit parameters $I_{\mathrm{s}}, R_{\mathrm{s}}, R_{\mathrm{p}}, n$ and $I_{\mathrm{p}}$ can now be utilized to quantitatively assess the local behavior of the fabricated SiNW device. The series resistance $R_{\mathrm{S}}$ is given by the sum of the resistance of the $\mathrm{W}$-needle contact, the internal resistance of the SiNW diode and the spreading resistance of the substrate wafer. The histogram (inset Fig. 5a) shows the extracted series resistances $R_{\mathrm{S}}$ retrieved from all 56 measurements/fits where $R_{\mathrm{S}}$ is sharply peaking at around $12.6 \Omega$. This implies that $R_{\mathrm{S}}$ is not significantly dependent on the minority carrier injection level. Therefore, contributions of the SiNW diode and the wafer to $R_{\mathrm{S}}$ can be neglected. Accordingly, $R_{\mathrm{s}}$ should mostly be determined by the mechanical contact between the SiNW and the W-needle which, in turn, is sufficiently small and stable to enable probing of the device current $I_{\mathrm{m}}$.

To quantify the probability of carriers to reach the $\mathrm{p}-\mathrm{n}$ junction in dependence on intensity $P$ and wavelength $\lambda$ of incident light, a photocarrier collection efficiency is defined as

$$
\operatorname{PCE}(\lambda, P)=\alpha \frac{j_{\mathrm{p}}}{j_{\hbar v}}
$$

where $j_{\mathrm{p}}$ is the photocurrent density in the individual diode, $j_{\hbar \nu}$ is the incident photon flux and $\alpha$ is a constant normalizing the maximum PCE value to 1. Fig. 4 shows the PCE a function of wavelength and intensity. It can be seen that it strongly decreases for lower wavelength and higher light intensity for which in the following two separate mechanisms will be made out. The lower PCE for higher illumination intensities has its origin in a smaller rectification of the SiNW diode for high illumination intensities and high photocurrent densities, $j_{\mathrm{p}}$.

Fig. 5 shows that the photocurrent density $j_{\mathrm{p}}$ for higher light intensities strongly rises, which should actually lead to an increase in PCE. Nevertheless, the PCE for high light intensities drops as Fig. 4 clearly shows. The reason can be found in deviations of the SiNW diode from a mostly carrier diffusion controlled ideal Shockley diode $\left(1 \leq n \leq 2, R_{\mathrm{p}} \rightarrow \infty, R_{\mathrm{S}} \approx 0\right.$ in eqn (1)). These deviations are most instructively described in terms of parameters $R_{\mathrm{p}}$ and $n$ of the equivalent circuit model (eqn (1)). According to Sah and Shockley, non-saturable reverse

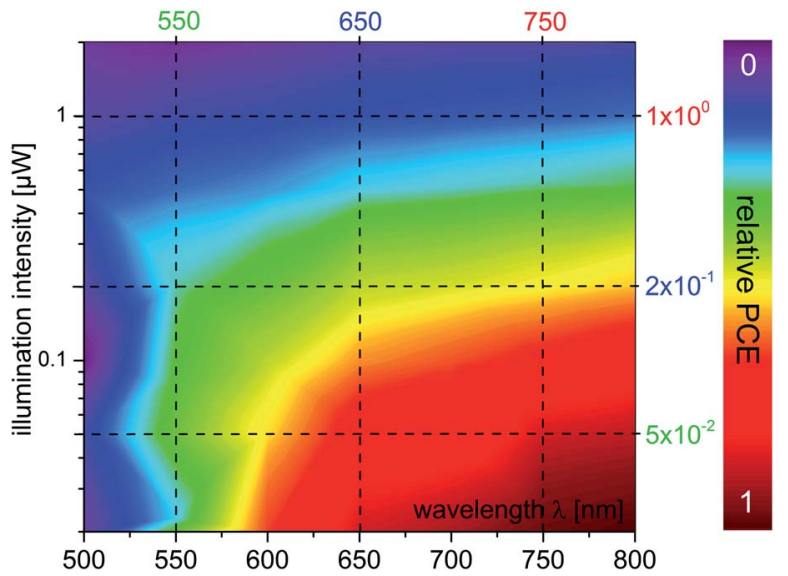

Fig. 4 Color coded relative PCE of the SiNW diode on the wafer substrate for different light wavelengths and intensities. Along dashed lines the diode photocurrent density $j_{\mathrm{p}}$, diode ideality factor $n$ and parallel/shunting resistivity $R_{\mathrm{p}}$ of the SiNW diode are plotted in Fig. 5 and 6.

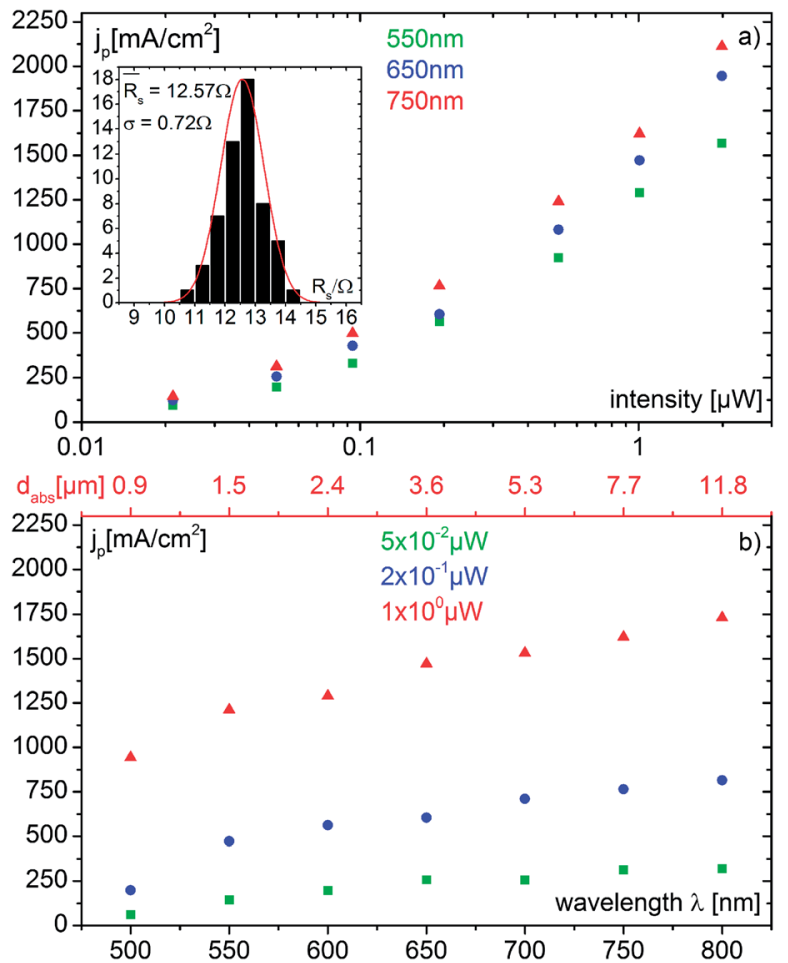

Fig. 5 Photocurrent density $j_{\mathrm{p}}$ in an individual SiNW diode as a function of intensity (three different wavelengths) and wavelength/mean absorption depth (three different intensities). ${ }^{21}$ The inset in the upper image shows a histogram over the series resistivity from all 56 measurements.

currents (i.e. $R_{\mathrm{p}}<\infty$ ) and a deviation of the forward bias behavior of a Si p-n diode from the ideal Shockley equation (i.e. $I \propto\left(\exp \left(\frac{e V}{n k T}\right)-1\right)$ with values $\left.n \leq 2\right)$ can be explained with the population of trap states in the depletion zone (space charge layer/simulations, Fig. 1). ${ }^{22}$ 
Fig. $6 \mathrm{a}$ and $\mathrm{b}$ show that the diode ideality $n$ and the shunting resistivity change significantly with increasing light intensity. $R_{\mathrm{p}}$ drops as the excitation level increases independently of the wavelength of incident light, indicating the enhanced population of trap states in the depletion zone for a high carrier injection in the device and high $j_{\mathrm{p}}$ in the SiNW diode (compare XPS scan of the as-etched surface, ESI $\dagger$ ). Axial SiNWs in general behave less ideal (i.e. have higher ideality factors $n$ ) in comparison with radial SiNWs. ${ }^{16,23}$ It has been speculated that this is due to the fact that in axial SiNW diodes the depletion region is exposed to the surface whereas in radial designs the depletion region is embedded within the SiNW itself. In the present case ideality factors $n$ are also quite high (between 5.5 and 6.5, Fig. 6a) and rise further for higher illumination intensities. Since the wafers used in the current study are high purity float zone $\mathrm{Si}$ it is most likely that high generation/ recombination currents (i.e. high quality factors $n$ ) stem from comparably rough and unpassivated surfaces of the SiNW (Fig. 1). As compared to planar devices, current densities in SiNW devices are typically higher due to the strong confinement of the current and the resulting current density in individual SiNW diodes will be a function of the surface filling fraction of contacted SiNWs. The current density of about $40 \mathrm{~mA} \mathrm{~cm}^{-2}$ for a planar silicon solar cell i.e. would lead to about $400 \mathrm{~mA} \mathrm{~cm}^{-2}$ for a $10 \%$ filling fraction of contacted SiNWs. ${ }^{24}$ In the presented case of an individual contacted diode, $j_{\mathrm{p}}$ in the SiNW diode gets as high as $2000 \mathrm{~mA} \mathrm{~cm}^{-2}$ corresponding to about $10^{19}$ electrons per s per $\mathrm{cm}^{2}$ in the diode, which is three orders of magnitude higher than the exciting photon flux density of about $10^{16}$ photons per $\mathrm{s}$ per $\mathrm{cm}^{2}$ ( $\sim 1 \mu \mathrm{W}$ from the fiber). Physically this means that the carriers contributing to $j_{\mathrm{p}}$ in the presented case emerge from an area at least 1000 times bigger than the SiNW. It can be concluded that SiNW diodes have to be designed in a way that they sustain a proper rectification especially at high current densities and injection levels. Further, the maximum reachable current density in a SiNW diode on a device can be controlled, in order to avoid the occurrence of the measured detrimental effects on diode rectification.

Lower photocurrent densities $j_{\mathrm{p}}$ flow through the SiNW diode for short wavelength light which is absorbed closer to the device surface. This is indicated in Fig. $5 \mathrm{~b}$ (top red axis) showing the absorption depth for the used different wavelengths. ${ }^{21}$ The results suggest that photocarriers which are generated close to the surface (i.e. within the SiNW array or directly below) have a lower probability to reach the $\mathrm{p}-\mathrm{n}$ junction i.e. are prone to surface recombination. Consequently, the PCE decreases for surface-near absorption, which makes it a measure for the effectiveness of the surface passivation of the SiNW array (compare Fig. 4). Fig. 6c and d show that $R_{\mathrm{p}}$ and $n$ remain constant for variations in light wavelength (three intensities selected for clarity), meaning that the rectification characteristics of the SiNW diode do not change for different wavelengths

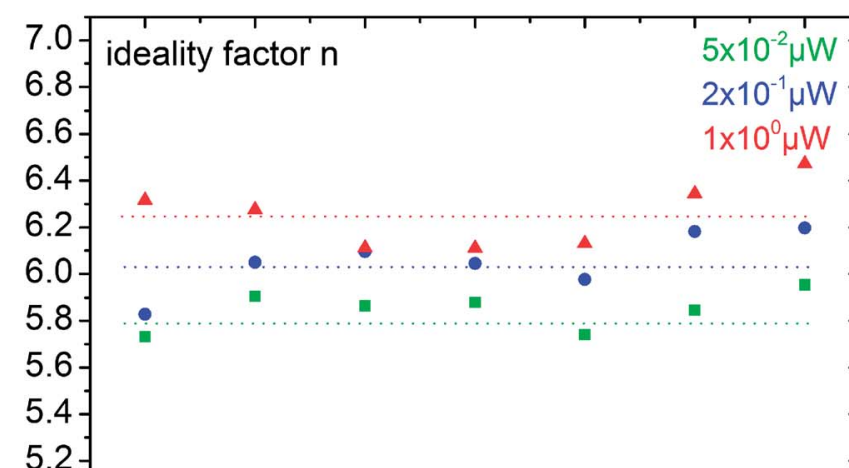

$5.0-$ a)

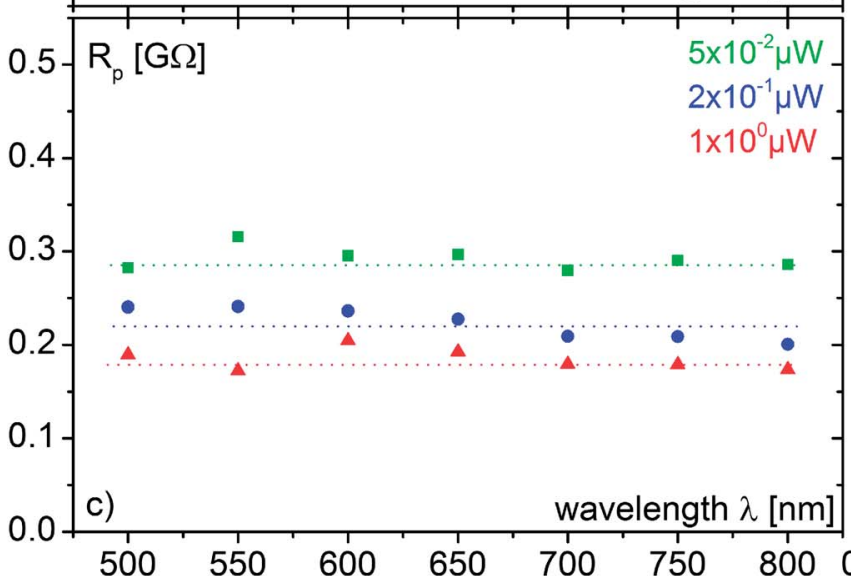

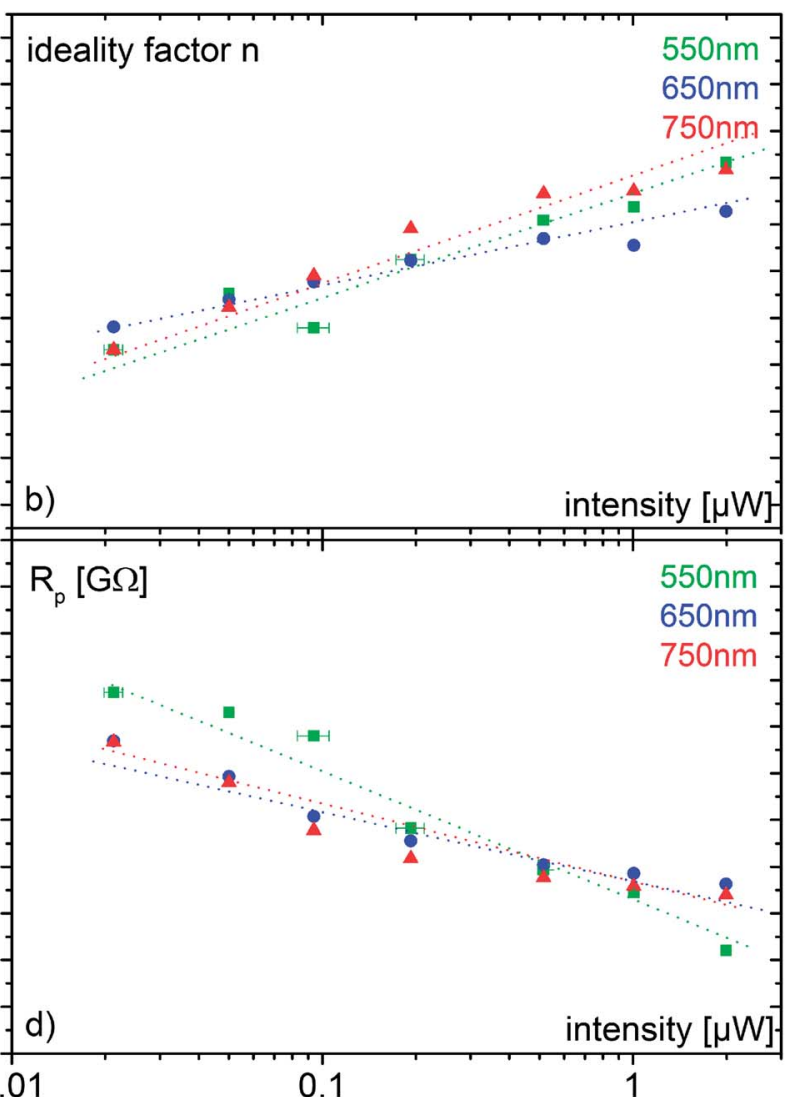

Fig. 6 SiNW diode ideality factor $n$ and parallel/shunting resistance $R_{\mathrm{p}}$ as a function of wavelength and intensity of incident light. Three intensities per wavelength/three wavelengths per intensity are plotted for clarity; dashed lines are a guide to the eye. 
of incident light. This confirms that the drop in PCE for low wavelengths is independent of the diode characteristics and is merely related to surface recombination.

\section{Conclusions}

An SEM-based nano-probing method is shown that permits the measurement of the dark and illuminated $I-V$ curves of an individual vertically aligned axial SiNW $\mathrm{p}-\mathrm{n}$ diode coupled to a wafer substrate. It turns out that the serially coupled system SiNW/wafer exhibits the characteristics of a single diode, and that the diode parameters like series and shunting resistivity, diode ideality factor and photocurrent can be retrieved from a fit of an equivalent circuit model. The determined PCE of the $\mathrm{p}-$ $\mathrm{n}$ junction in the SiNW allows a quantitative assessment of various design aspects for nano-enabled devices. We find that the PCE strongly depends on the absorption depth (wavelength) of the incident light which allows quantification of the effectiveness of the device surface passivation at the nanoscale. Further, it turns out that high photo-carrier densities predominantly emerging from the wafer substrate reduce the rectification of the individual SiNW diode, which leads to a reduction of PCE for high injection levels. This is especially relevant for nanowire enabled device designs where photocurrent densities exceed the values for planar devices.

\section{Acknowledgements}

S.W.S., G.B., F.S., M.B. and S.C. acknowledge partial financial support by the FP7 Projects FIBLYS (grant: 214042), Univsem (grant: 280566) and Lcaos (grant: 258868) and the BMBF project Nawion (FKZ: 16SV5386K, V4MNI014). S.K.S. thanks the Department of Science and Technology, Government of India for BOYSCAST fellowship (Award no. SR/BY/P-03/10).

\section{Notes and references}

1 Y. Cui, X. Duan, J. Hu and C. M. Lieber, J. Phys. Chem. B, 2000, 104, 5213-5216.

2 Y. Cui and C. M. Lieber, Science, 2001, 291, 851-853.

3 P. Alivisatos, Nat. Biotechnol., 2004, 22, 47-52.

4 G. Zheng, F. Patolsky, Y. Cui, W. U. Wang and C. M. Lieber, Nat. Biotechnol., 2005, 23, 1294-1301.

5 Y. Cui, Z. Zhong, D. Wang, W. U. Wang and C. M. Lieber, Nano Lett., 2003, 3, 149-152.
6 W. M. Weber, L. Geelhaar, A. P. Graham, E. Unger, G. S. Duesberg, M. Liebau, W. Pamler, C. Chèze, H. Riechert, P. Lugli and F. Kreupl, Nano Lett., 2006, 6, 2660-2666.

7 E. C. Garnett and P. Yang, J. Am. Chem. Soc., 2008, 130, 92249225.

8 V. Sivakov, G. Andrä, A. Gawlik, A. Berger, J. Plentz, F. Falk and S. H. Christiansen, Nano Lett., 2009, 9, 1549-1554.

9 L. Tsakalakos, J. Balch, J. Fronheiser, B. A. Korevaar, O. Sulima and J. Rand, Appl. Phys. Lett., 2007, 91, 233117.

10 D. D. D. Ma, C. S. Lee, F. C. K. Au, S. Y. Tong and S. T. Lee, Science, 2003, 299, 1874-1877.

11 R. Q. Zhang, Y. Lifshitz, D. D. D. Ma, Y. L. Zhao, T. Frauenheim, S. T. Lee and S. Y. Tong, J. Chem. Phys., 2005, 123, 144703.

12 G. Brönstrup, N. Jahr, C. Leiterer, A. Csaki, W. Fritzsche and S. Christiansen, ACS Nano, 2010, 4, 7113-7122.

13 L. Cao, P. Fan, A. P. Vasudev, J. S. White, Z. Yu, W. Cai, J. Schuller, S. Fan and M. L. Brongersma, Nano Lett., 2010, 10, 439-445.

14 S.-W. Chung, J.-Y. Yu and J. R. Heath, Appl. Phys. Lett., 2000, 76, 2068-2070.

15 B. Tian, X. Zheng, T. J. Kempa, Y. Fang, N. Yu, G. Yu, J. Huang and C. M. Lieber, Nature, 2007, 449, 885-889.

16 J. D. Christesen, X. Zhang, C. W. Pinion, T. a. Celano, C. J. Flynn and J. F. Cahoon, Nano Lett., 2012, 12, 6024-6029.

17 H. J. Leamy, J. Appl. Phys., 1982, 53, R51.

18 S. W. Schmitt, F. Schechtel, D. Amkreutz, M. Bashouti, S. K. Srivastava, B. Hoffmann, C. Dieker, E. Spiecker, B. Rech and S. H. Christiansen, Nano Lett., 2012, 12, 40504054.

19 M. Galassi, J. Davies, J. Theiler, B. Gough, G. Jungman, M. Booth and F. Rossi, Gnu Scientific Library Reference Manual, 2009, p. 592.

20 W. Shockley, Bell Syst. Tech. J., 1949, 28(3), 435-489.

21 M. A. Green and M. J. Keevers, Prog. Photovoltaics, 1995, 3, 189-192.

22 C. Sah, R. N. Noyce and W. Shockley, Proc. IRE, 1957, 45, 1228-1243.

23 T. J. Kempa, B. Tian, D. R. Kim, J. Hu, X. Zheng and C. M. Lieber, Nano Lett., 2008, 8, 3456-3460.

24 S. W. Glunz, J. Knobloch, C. Hebling and W. Wettling, Conference Record: Twenty Sixth IEEE Photovoltaic Specialists Conference, 1997. 